\title{
A NEW PARALLEL HYBRID MULTIOBJECTIVE ANT COLONY ALGORITHM BASED ON OPENMP
}

\author{
Imen Ben Mansour ${ }^{2,1}$, Ines Alaya ${ }^{1}$ and Moncef Tagina ${ }^{1}$ \\ IENSI-COSMOS, University of Manouba, Manouba 2010, Tunisia \\ ${ }^{2}$ Esprit School Of Engineering, Tunis, Tunisia
}

\begin{abstract}
Parallel computing constitutes a growing area of interest in solving many complex combinatorial optimization problems. This paper presents a parallel implementation of a hybrid ant colony optimization metaheuristic for the multiobjective knapsack problem using the OpenMP framework. The hybrid algorithm coupled a MultiObjective Ant Colony Optimization (MOACO) algorithm with Tchebycheff based Local Search (TLS) procedure. The parallelization main idea is defined as assuming a shared-memory based on threads in which the initialization phase begins with a single thread called the master thread and executed sequentially. Afterward, a parallel region is defined where many threads are created, each one of them executing its own copy of the proposed ant colony algorithm independently. The threads cooperate through sharing a global archive holding all non-dominated solutions found so far. Experimental results show a significant efficiency of the solutions returned over the sequential implementation.
\end{abstract}

\section{KEYWORDS}

Parallel Metaheuristic, Threads, OpenMP, Ant Colony Optimization, Multiobjective Optimization, The Augmented Weighted Tchebycheff Method

\section{INTRODUCTION}

Several real-life optimization problems are modeled as a multiobjective combinatorial optimization problem, due to the multiple conflicting objectives and the different constraints that have to be respected simultaneously. Since such optimization problems cannot be tackled by exacts methods, metaheuristics represent a great alternative, they are used with less computational effort to find a good approximation of the optimal Pareto set.

Since introduced in 1992 by Marco Dorigo (Dorigo, 1992), ant colony optimization (ACO) algorithms have been successfully applied to many combinatorial mono and multi-objective optimization problems, ranging from traveling salesmen (TSP) (Angus, 2007; Dorigo \& Gambardella, 1991) to knapsack problems (KP) (Alaya, Solnon, \& Ghédira, 2007; Zouari, Alaya, \& Tagina, 2017) and a lot of derived methods have been adapted to cooperative and parallel implementations.

ACO was proposed as a solution when suffering from limited diversification. Therefore, ACO is a powerful technique in exploration of the solution space. In addition to its significant performance improvement compared with other metaheuristic techniques, its multicore computing power encouraged the modification of the standard sequential form to be applied in a parallel framework.

In this paper, we propose an efficient and straightforward OpenMP multiobjective ACO (MOACO) implementation based on shared memory multiprocessing programming architecture. Here, OpenMP is implemented with its parallel regions. The proposed framework begins as a single execution thread called the master thread. When the thread meets the parallel sections, it creates a team of threads consisting of the initial thread itself and the other threads and becomes the main thread in the team. All the members of the team execute a copy of the MOACO algorithm. At the end of the parallel sections, the further execution of the code is performed only by the master thread. Therefore, the main thread is responsible for the update the global archive holding all the non-dominated solutions found so far. The design of a parallel framework is motivated by, firstly, the need to improve the convergence and the diversity of the solutions, secondly to reduce computing efforts without altering its behavior. 
This paper is organized as the following: in Section 2, the related work to ACO and the research efforts towards its parallelization are presented. Section 3 introduces the studied problem: the multiobjective KP. Section 4 presents the sequential ACO algorithm mapped to the multiobjective knapsack problem. In section 4, the proposed ACO parallelization using OpenMP is introduced where its sub-sections show the analysis of different elements of OpenMP and its effects on performance. In section 5, results and performance evaluation are investigated using the multiobjective KP problem as an implementation of the parallel ACO algorithm. Finally, section 6 concludes the research and suggests the future work.

\section{RELATED WORKS}

Many efforts have been devoted to adapting the ACO algorithm on several cooperative and parallel paradigms (Pedemonte, Nesmachnow, \& Cancela, 2011). The first implementation of a parallel Ant System is attributed to (Bolondi \& Bondanza, 1993), who presented a fine-grain implementation that uses several groups of ants executed a standard AS algorithm and placed one ant in each processor to solve the Traveling Salesman Problem (TSP).

Talbi et al. in (Talbi, Roux, Fonlupt, \& Robillard, 2001) presented a method called ANTabu combining ACO and Tabu Search (TS), applied to solve the Quadratic Assignment Problem (QAP). The TS method was used as a local search to improve the solutions in each thread. The parallel version was compared against a sequential ACO, a parallel TS, genetic algorithms (GA) and variable neighborhood search. In (Lv, Xia, \& Qian, 2006) Lv et al. proposed a parallel ACO using P groups of ants, distributed in P processors, which shared one pheromone matrix in multiprocessing computer to solve TSPLIB instances with up to 15,915 cities. The algorithm achieved better solutions than sequential versions. Stützle in (Stützle \& Hoos, 2000) studied the parallel independent execution of $\mathcal{M} \mathcal{A} \mathcal{X}-\mathcal{M} \mathcal{N}$ Ant System ( $\mathcal{M} \mathcal{M}$ AS) with a local search for the TSP. The evaluation analyzed the benefits of using a parallel model with respect to a sequential ACO, comparing the quality of the solutions for TSPLIB instances with up to 1173 cities. The results showed that the parallel executions obtained better solutions than the sequential algorithm in all the studied instances. In (Bai, OuYang, Li, He, \& Yu, 2009), the authors implemented parallel independent runs of MMAS on GPU. Each thread executed one ant and each thread block was used for independent execution. The main algorithm runs on GPU, while the CPU is only used to initialize the solutions and to control the iteration process. Regarding the quality of the solutions, the GPU-parallel implementation outperformed three sequential MMAS versions, while acceleration values between 2 and 32 were obtained. In (Abouelfarag, Aly, \& Elbialy, 2015), the authors proposed a parallel ACO using the OpenMP framework. Parallel regions are used, to increase the overhead of creating and terminating threads and a shared memory model has been chosen to get the benefit of creating a common space sharing pheromone matrix without the overhead of communication.

Although several studies have been proposed for the parallel implementation of mono-objective ACO, as far as we know, few references can be found about parallel implementations of the multiobjective ACO (MOACO) (Falcón-Cardona, Leguizamón, Coello, \& Tapia, 2020), even less with the openMP framework. In (Delisle, Krajecki, Gravel, \& Gagné, 2001) a parallel implementation of an ant colony optimization metaheuristic with OpenMP for the solution of an industrial multiobjective scheduling problem in an aluminum casting center is introduced. The parallelization process consists to affect the generation and evaluation of each ant to a different processor. The pheromone matrix is in the shared memory and is updated by one ant, by an OpenMP thread, at a time, in a critical section of the parallel region. In (Mora, García-Sánchez, Merelo, \& Castillo, 2013), the authors introduce an island-based model where the colonies communicate by migrating ants, following a neighborhood topology that fits the search space. Thus a parallel scheme will be implemented, taking a coarse-grained parallelization approach, at the colony level, so every computational node (processor) will contain a set of ants (colony).

This paper proposes a solution with OpenMP to get the performance gain of parallel regions. These parallel regions provide parallelizing to the MOACO algorithm not only to speed-up the MOACO but also to improve its Pareto set's quality. 


\section{MULTIOBJECTIVE KNAPSACK PROBLEM}

The multiobjective multidimensional knapsack problem consists of finding a subset of items subject to a set of resource constraints while maximizing several objectives. Due to its NP-hard nature (Martello, 1990), several approaches have been proposed (Lust, \& Teghem, 2012). Formally, MOMKP could be written as follows:

$$
\begin{array}{rrr}
\text { Maximize } \quad \sum_{j=1}^{n} p_{j}^{k} x_{j} & k=1, \ldots, m \\
\text { Subject to } & \sum_{j=1}^{n} w_{j}^{i} x_{j} \leq b_{i} & i=1, \ldots, q \\
x_{j} \in\{0,1\} & j=1, \ldots, n
\end{array}
$$

There are $n$ items, for each item $I_{j}$ is assigning a decision variable $x_{j}$ equal to 1 if the item is selected, 0 otherwise. Each item $I_{j}$ has a profit $p_{j}^{k}$ relatively to the objective $k$ and $w_{i j}$ consumed quantity relative to the resource $i$ (like weight, volume...). The aim objective of the problem is to select a subset of items among the $n$ items in order to maximize $m$ objective functions while not exceeding $q$ resource constraints $b_{i}$ regarding the total quantity available for the resource $i$.

\section{SEQUENTIAL HYBRID MULTIOBJECTIVE ANT COLONY OPTIMIZATION FOR MOMKP}

ACO metaheuristic is a cooperative population-based construction algorithm inspired from the behavior of real ants while searching for a food source. The colony of ants cooperates to build a set of solutions using an indirect form of communication, called pheromone, deposited by the member of the colony while building their solutions.

The Tchebycheff-based Local Search (TLS) procedure is a simple heuristic algorithm used to find good-quality solutions or to improve results in a relatively short time. They provide an interesting alternative to classical evolutionary algorithms and often involve a small number of parameters.

Here, the hybrid approach is proposed as a synergy of the multiobjective ACO algorithm with a Tchebycheff-based Local Search (TLS) procedure. The MOACO algorithm is used to favor the exploration in the search space while the TLS method tries to enhance the solutions built by ants. Since our algorithm follows the MMAS scheme (Stützle \& Hoos, 2000) so, as a first step, the pheromone traces are initialized to an upper bound $\tau$ max. Apart from that, mainly, the hybrid MOACO algorithm for MOMKP consists of three procedures described in the following sub-sections.

\subsection{Construct Ants Solutions}

To tailor the ACO for solving the Knapsack problem (KP), the problem must be reduced to a complete graph $\mathrm{G}=(\mathrm{V}, \mathrm{E})$ along which the ants move to build candidate solutions. Where $\mathrm{V}$ is the set of vertices and $\mathrm{E}$ is the set of edges. The vertices $\mathrm{V}$ acted as the set of items to be selected and E represents the connection between the vertices $\mathrm{V}$ i.e. path constructed by a given ant. Here, the pheromone trails are associated with the vertices of this graph. Each ant $\mathrm{h}$ constructs one feasible solution by applying repeatedly the state transition rule to select the most appropriate item $\mathrm{I}_{\mathrm{j}}$ to be added to the solution $\mathrm{S}^{\mathrm{h}}$ among a set of feasible items Feas (candidate vertices). This set is updated by including items not yet added and that doesn't violate any constraint. The transition rule $\mathrm{p}_{\mathrm{S}}^{\mathrm{h}}$ is directed by the pheromone value $\tau_{\mathrm{S}}{ }^{\mathrm{h}}$ and the heuristic information $\eta_{\mathrm{S}} \mathrm{h}$ :

$$
\mathrm{p}_{\mathrm{S}^{\mathrm{h}}}\left(\mathrm{I}_{\mathrm{j}}\right)=\frac{\left[\tau_{\mathrm{S}^{h}}\left(\mathrm{I}_{\mathrm{j}}\right)\right]^{\alpha}\left[\eta_{\mathrm{S}^{h}}\left(\mathrm{I}_{\mathrm{j}}\right)\right]^{\beta}}{\sum_{\mathrm{I}_{\mathrm{l}} \in \text { Feas }}\left[\tau_{\mathrm{sh}^{h}}\left(\mathrm{I}_{\mathrm{l}}\right)\right]^{\alpha}\left[\tau_{s^{h}}\left(\mathrm{I}_{\mathrm{l}}\right)\right]^{\alpha}}
$$

The heuristic information is used to guide the search process of artificial ants. In order to orientate ants to look in different regions of the non-dominated front, different configurations of the heuristic information matrix are executed, i.e. using different weight vectors $\lambda(g)=\left(\lambda_{1}(g), \ldots, \lambda_{m}(g)\right)$. To generate the set of the weight vector, we use the Gradual weights generation method (Gw) (Mansour, Alaya, \& Moncef, 2017a, $2017 b, 2019)$. For that, $\eta_{s}{ }^{h}$ for a given ant $h$, is set as: 


$$
\eta_{\mathrm{S}}^{\mathrm{h}}\left(\mathrm{I}_{\mathrm{j}}\right)=\frac{\sum_{\mathrm{k}=1}^{\mathrm{m}} \lambda_{\mathrm{k}}(\mathrm{g}) \mathrm{p}_{\mathrm{j}}^{\mathrm{k}}}{\sum_{\mathrm{i}=1}^{\mathrm{q}}\left(\frac{\mathrm{w}_{\mathrm{j}}^{\mathrm{i}}}{\mathrm{R}_{\mathrm{S}}^{\mathrm{h}}(\mathrm{i})}\right)}
$$

where is the remaining amount of the resource $\mathrm{i}$ when an ant $\mathrm{h}$ is currently building its solution $S^{\mathrm{h}} \cdot \mathrm{p}^{\mathrm{k}} \mathrm{j}$ and $\mathrm{w}_{\mathrm{j}}^{\mathrm{i}}$ are respectively the profit and the weight of the candidate item.

\subsection{Local Search}

This step is started after the solution construction phase and before the pheromone update. The local search procedure aims to improve the quality of solutions generated by the Ant Colony algorithm. The fitness value of each solution in Sol is calculated before performing a local search step. This step consists of exploring the neighborhood of each solution $S^{\mathrm{h}}$ in Sol until we find a solution $\mathrm{S}^{\mathrm{h}^{*}}$ that is better than the worst solution w of Sol regarding the search direction $\lambda$ under consideration. Then, $S^{\mathrm{h}^{*}}$ is added to Sol and replaces the solution $\mathrm{w}$. The neighborhood exploration process stops once the first improving neighbor is found.

Since the 0/1 multi-objective knapsack problem is a constrained problem, all solutions should satisfy all resource constraints. Indeed, to generate a new neighbor of $S^{h}$, we have to use an efficient neighborhood structure for this problem. To this end, we use the same neighborhood function proposed in (Mansour, Alaya, \& Moncef, 2017a). This function allows establishing an order between items according to their profitability. It has the goal of finding the item that minimizes an extraction ratio to permute it with items that maximize an insertion ratio in order to improve the quality of the obtained solutions, i.e. the neighbor in a small computational time.

In order to evaluate a solution $\mathrm{S}^{\mathrm{h}}$ against the whole population, we use one of the most commonly-used scalarization approaches: the augmented weighted Tchebycheff method (Steuer \& Choo, 1983).

\subsection{Pheromone Update}

The pheromone update step is the most important phase. When all ants construct their solutions, the non-dominated solutions of the current generation are stored in the archive. The ants that are allowed to update the pheromone trails, are thus who have found the member of the current archive by laying an amount of pheromone on each item. The pheromone trail is updated as follows:

$$
\tau\left(I_{j}\right) \leftarrow(1-\rho) * \tau\left(I_{j}\right)+\Delta \tau\left(I_{j}\right)
$$

with

$$
\Delta \tau\left(I_{j}\right)=\left\{\begin{array}{l}
|A| \text { if } I_{j} \in S_{N D} \\
0 \quad \text { otherwise }
\end{array}\right.
$$

In order to give every generation of ants the same influence in a particular part of the Pareto front, ants that are authorized to update, lay the same amount of pheromone which is equal to the current archive set size.

\section{THE PROPOSED HYBRID MOACO PARALLELIZATION BASED ON OPENMP}

The Ant Colony Optimization is a potential candidate for parallelization for different reasons. One can cite: The large number of iterations required in updating pheromone trails, the computations needed for the single ant/group of ants to construct solution(s) and the independent behavior of ants.

Many strategies have been proposed to implement a parallel ACO. In this paper, we adopt a parallelization strategy based on multi-thread programming with multi-core processors. This section introduces an implementation for parallel MOACO using the OpenMP platform. A shared memory model 
has been considered to get the benefit of creating a common space sharing pheromone matrix and Pareto sets without the overhead of communication, especially when applying both the Construct Ant Solutions and Update Pheromone processes. The proposed approach is implemented to improve the approximation of the Pareto-optimal solutions not altering the MOACO algorithm behavior.

The idea is to execute several algorithms instead of a panmictic one, since the management of the many populations has a great impact on the efficiency of the approach and its ability to compute a sufficiently diverse and representative Pareto front. Also, we choose to parallel the places which consume most execution time in the sequential MOACO and to overcome the problem of communication overhead by using the OpenMP directives. Larger OpenMP parallel regions are used, because fragmented parallel regions would increase the overhead of creating and terminating threads. Figure 1. shows the parallel programming method of the proposed Algorithm, Multiobjective Hybrid Ant Colony based on OpenMP (MHAC_OMP). The algorithm is divided into four sections relatively to the "sections" of OpenMP. Each section is assigned to one thread. Once all threads finish the execution of MHAC_OMP, the shared global archive P is updated and returned.

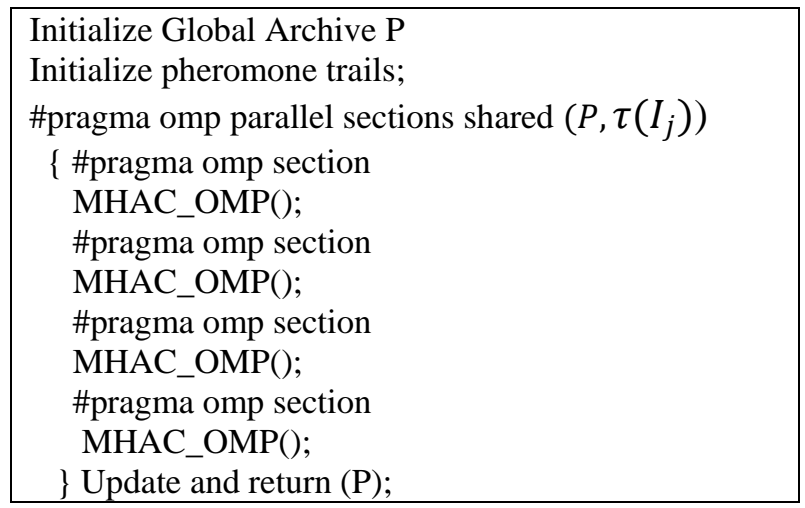

Figure 1. Parallel Programming method of MHAC_OMP

In summary, the structure of the MHAC_OMP algorithm is given in Figure 2. The left side of the figure describes the MHAC_OMP algorithm while the right side presents the local search procedure used in the proposed MHAC_OMP algorithm.

\begin{tabular}{|c|c|}
\hline 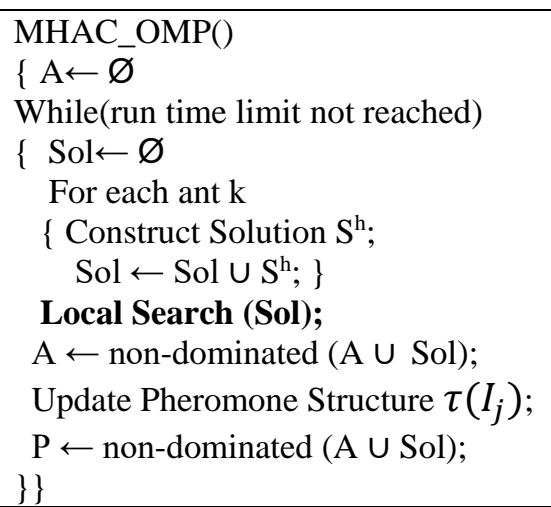 & 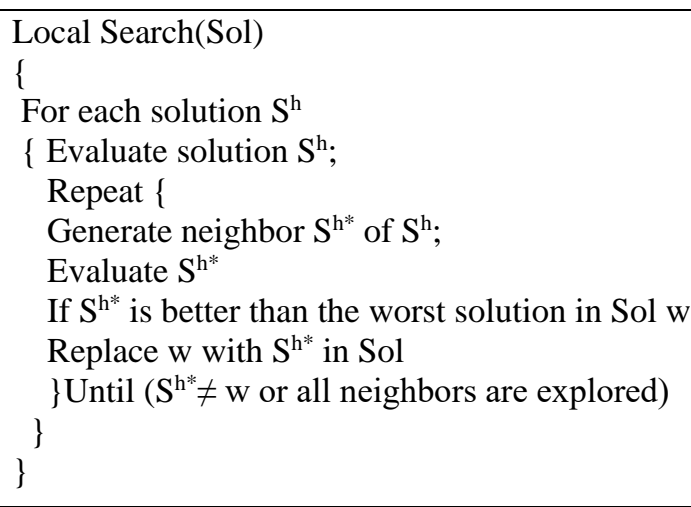 \\
\hline
\end{tabular}

Figure 2. The MHAC_OMP algorithm (left side) and the local search procedure (right side)

\section{EXPERIMENTS AND RESULTS}

In this section, we assess the performance of the MHAC_OMP. The platform for conducting the experiments is a IntelCore i5 $2.60 \mathrm{GHz}$ laptop with $6 \mathrm{~GB}$ RAM. The program was implemented using C++ language with OpenMP 4.0. We examine the performance of MHAC_OMP on nine instances of MOMKP (Zitzler 
\& Thiele, 1999) with two sequential MOACO algorithms: Gw-ACO (Mansour, Alaya, \& Moncef, 2019) and the sequential version of MHAC_OMP called here MHAC.

To evaluate the efficiency of MHAC_OMP, we use as metrics of performance the hypervolume difference (HypD) (Zitzler \& Thiele, 1999). The HypD value has to be as close as possible to zero to prove the efficiency of the algorithm. We use also the summary attainment surface (Da Fonseca, Fonseca, \& Hall, 2001), to compare the approaches behaviors in a graphical way. We perform the non-parametric Mann-Whitney statistical test described in (Knowles, Thiele, \& Zitzler, 2005.) in order to verify if the difference between the tested algorithms is statistically significant with a confidence level greater than $95 \%$ ( $\mathrm{p}$-value $\leq 0.05$ ). The bold values in the tables denote the best results found for the considered instance by the corresponding algorithm.

\subsection{Parametrization of MHAC_OMP}

Metaheuristic algorithms and parallel approaches require a crucial decision about the values of numerous parameters. The solution quality and speed may be affected by the parameter settings. Accordingly, we have been striven to determine an appropriate set of parameter values for the MD-HACO. The number of threads is fixed to 4 , the significance weights for pheromone trail $\alpha$ set to 1 , the significance weights for heuristic information $\beta$ set to 10 , pheromone evaporation rate $\rho$ set to 0.90 , the number of ants equal to 10 , the lower bound of pheromone $\tau_{\min }$ is 1 and the upper bound $\tau_{\max }$ is 5 .

Each algorithm runs 30 times and each time we run 100 seconds if the number of objectives if 2, 300 seconds if is equal to 3 and 500 seconds with 4 objectives. We use for each algorithm the same computation time budget. This choice was motivated by the fact that measuring the speed up using the time taken by parallel and sequential algorithms to find good solutions is a more significant measure of performance than simply comparing the time taken to run a certain number of generations.

\subsection{Experimental Results}

Table 1 reports the average values obtained by the hypervolume difference metric when comparing MHAC_OMP, Gw-ACO and MHAC. By analyzing the table it is clear that MHAC_OMP achieves the best results. In fact, MHAC_OMP is more effective than Gw-ACO. Moreover, this parallel approach returns better values than the sequential one on all tested instances.

Table 2. Average values of the hypervolume difference metric of MHAC_OMP, Gw-ACO and MHAC

\begin{tabular}{llll}
\hline Instance & MHAC_OMP & Gw-ACO & MHAC \\
\hline $250 \_2$ & $\mathbf{1 . 5 0 E - 0 1}$ & $4.29 \mathrm{E}-01$ & $2.32 \mathrm{E}-01$ \\
$500 \_2$ & $\mathbf{1 . 3 9 E - 0 1}$ & $4.28 \mathrm{E}-01$ & $1.92 \mathrm{E}-01$ \\
$750 \_2$ & $\mathbf{1 . 2 8 E - 0 1}$ & $4.20 \mathrm{E}-01$ & $1.45 \mathrm{E}-01$ \\
$250 \_3$ & $\mathbf{1 . 6 9 E - 0 1}$ & $5.41 \mathrm{E}-01$ & $1.70 \mathrm{E}-01$ \\
$500 \_3$ & $\mathbf{1 . 8 0 E}-01$ & $5.49 \mathrm{E}-01$ & $2.02 \mathrm{E}-01$ \\
$750 \_3$ & $\mathbf{1 . 4 5 E - 0 1}$ & $5.72 \mathrm{E}-01$ & $1.81 \mathrm{E}-01$ \\
$250 \_4$ & $\mathbf{1 . 7 3 E - 0 1}$ & $5.59 \mathrm{E}-01$ & $2.12 \mathrm{E}-01$ \\
$500 \_4$ & $\mathbf{1 . 4 9 E - 0 1}$ & $5.23 \mathrm{E}-01$ & $1.77 \mathrm{E}-01$ \\
$750 \_4$ & $\mathbf{1 . 4 9 E - 0 1}$ & $5.60 \mathrm{E}-01$ & $1.76 \mathrm{E}-01$ \\
\hline
\end{tabular}

Table 2 gives the p-values of the Mann-Whitney statistical test of MHAC_OMP against Gw-ACO and MHAC. Indeed, this table confirms the results obtained in Table 1. The returned values highlight that MHAC_OMP strongly outperforms Gw-ACO on all tested instances. When comparing the p-values obtained by MHAC_OMP against MHAC, one can say that the parallel approach statistically outperforms the sequential one in almost all instances. In fact, MHAC_OMP is significantly better than MHAC for 7 out of the 9 instances. Moreover, it seems that the results of the parallel algorithm increase according to the size of the problem. Clearly, the larger the instance is, the better the results are.

Figure 3 plots the median attainment surface of the approximation sets of MHAC_OMP, Gw-ACO and MHAC on bi-objective instances. The figure shows that MHAC_OMP produces a very well-distributed Pareto front. It is evident from the figure 3, that almost all the final solutions obtained by MHAC_OMP dominate those obtained by $\mathrm{Gw}-\mathrm{ACO}$ and there are no solutions returned by Gw-ACO that dominate anyone returned by MHAC_OMP and this is for all the bi-objective tested instances. 
Table 2. The p-value of the Mann-Whitney statistical test of MHAC_OMP compared to Gw-ACO and MHAC

\begin{tabular}{lll}
\hline Instance & Gw-ACO & MHAC \\
\hline $250 \_2$ & $\leq \mathbf{0 . 0 5}$ & $\leq \mathbf{0 . 0 5}$ \\
$500 \_2$ & $\leq \mathbf{0 . 0 5}$ & $\leq \mathbf{0 . 0 5}$ \\
$750 \_2$ & $\leq \mathbf{0 . 0 5}$ & 0.38 \\
$250 \_3$ & $\leq \mathbf{0 . 0 5}$ & 0.5 \\
$500 \_3$ & $\leq \mathbf{0 . 0 5}$ & $\leq \mathbf{0 . 0 5}$ \\
$750 \_3$ & $\leq \mathbf{0 . 0 5}$ & $\leq \mathbf{0 . 0 5}$ \\
$250 \_4$ & $\leq \mathbf{0 . 0 5}$ & $\leq \mathbf{0 . 0 5}$ \\
$500 \_4$ & $\leq \mathbf{0 . 0 5}$ & $\leq \mathbf{0 . 0 5}$ \\
$750 \_4$ & $\leq \mathbf{0 . 0 5}$ & $\leq \mathbf{0 . 0 5}$ \\
\hline
\end{tabular}

From the same figure, one can see that the surfaces of MHAC_OMP and those of MHAC are difficult to distinguish visually, but it is clear that MHAC_OMP returns an important number of solutions than MHAC and that the parallel approach provides a better distribution i.e. the surfaces of MHAC_OMP cover all the extreme ends of the Pareto front.
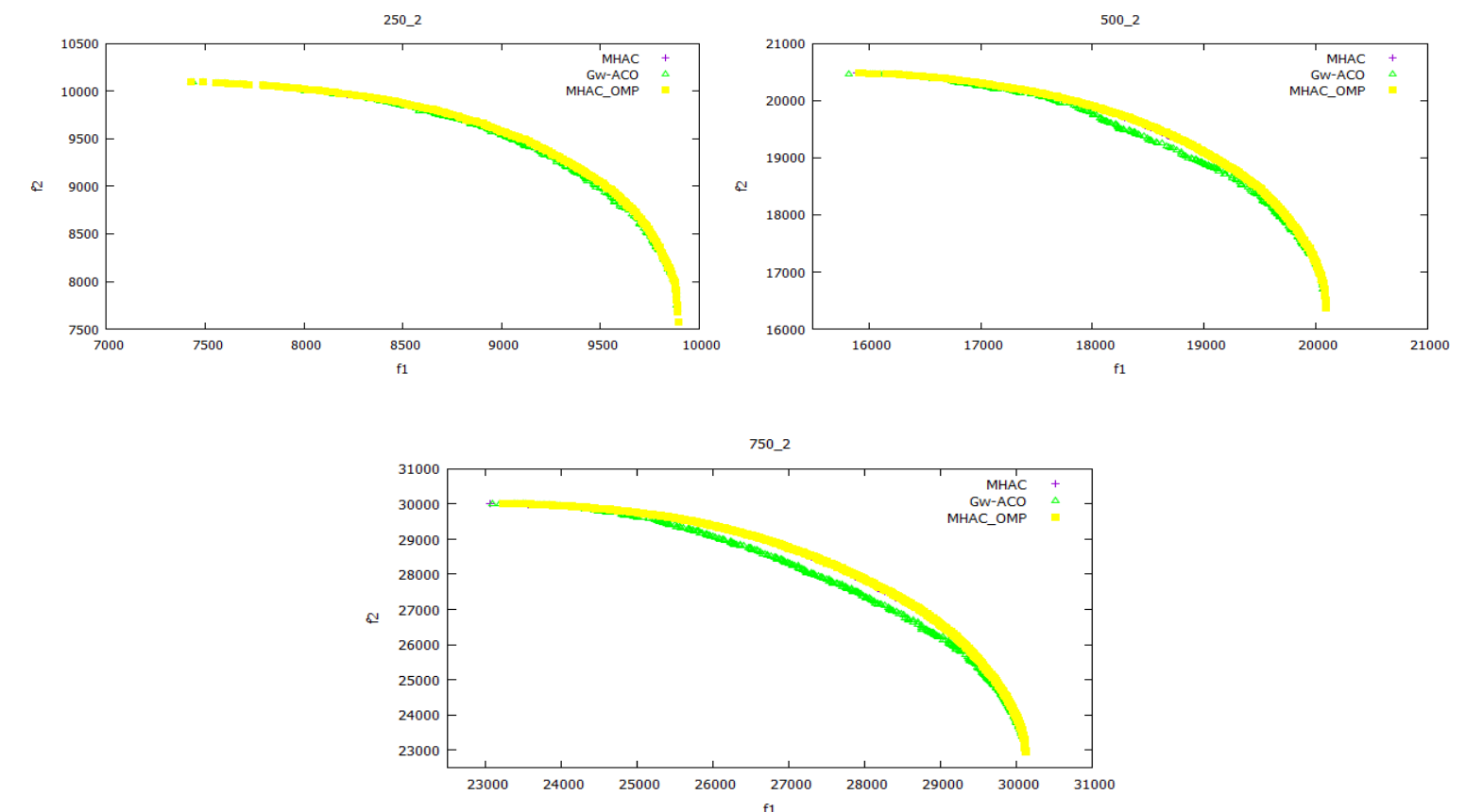

Figure 3. The median attainment surface obtained by MHAC_OMP, Gw-ACO and MHAC on bi-objective instances

\section{CONCLUSION}

In this paper, we have proposed a parallel Multiobjective Ant Colony Optimization approach based on OpenMP to solve the Multiobjective Knapsack Problem. The nature of the ACO and the functionality offered by OpenMP made the transition from sequential to parallel easier and straightforward, while some modifications had to be made to the algorithm to obtain the level of efficiency that we achieved in comparison with other approaches. Ongoing work follows two main directions: the study of the further properties of OMP with MOACO and the development of further applications to multiobjective combinatorial optimization problems. 


\section{REFERENCES}

Abouelfarag, A. A., Aly, W. M., \& Elbialy, A. G., 2015. Performance analysis and tuning for parallelization of ant colony optimization by using OpenMP. In IFIP International Conference on Computer Information Systems and Industrial Management. Springer, Cham. 73-85.

Alaya, I., Solnon, C., \& Ghédira, K., 2007. Ant Colony Optimization for Multi-objective Optimization Problems. 19th IEEE International Conference on Tools with Artificial Intelligence (ICTAI'07), 450-457.

Angus, D, 2007. Crowding population-based ant colony optimisation for the multi-objective travelling salesman problem. IEEE Symposium on Computational Intelligence in Multi-Criteria Decision-Making (MCDM),333-340.

Bai, H., OuYang, D., Li, X., He, L., \& Yu, H., 2009. Max-min ant system on gpu with cuda. Proceedings of the 2009 Fourth International Conference on Innovative Computing, Information and Control, IEEE Computer Society. 801-804.

Bolondi, M., \& Bondanza, M., 1993. Parallelizzazione di un algoritmo per la risoluzione del problema del commesso viaggiatore (Doctoral dissertation, Master's thesis, Dipartimento di Elettronica e Informazione, Politecnico di Milano, Italy).

Delisle, P., Krajecki, M., Gravel, M., \& Gagné, C., 2001. Parallel implementation of an ant colony optimization metaheuristic with OpenMP. Proceedings of the 3rd European Workshop on OpenMP (EWOMP'01), Barcelona, Spain.

Dorigo M., 1992. Optimization, Learning and Natural Algorithms. PhD thesis. Politecnico di Milano, Italy.

Dorigo, M., \& Gambardella, L. M., 1997. Ant colony system: A cooperative learning approach to the traveling salesman problem. Evolutionary Computation, IEEE Transactions, 1(1), 53-66.

Falcón-Cardona, J. G., Leguizamón, G., Coello, C. A. C., \& Tapia, M. G. C., 2020 Multi-Objective Ant Colony Optimization: An Updated Taxonomy and Review of Approaches.

Da Fonseca, VG., Fonseca, CM., \& Hall, AO., 2001. Inferential performance assessment of stochastic optimisers and the attainment function. 1st International Conference on Evolutionary Multi-criterion Optimization (EMO 2001). Lecture Note in Computer Science. 1993,213-225. Springer, Berlin.

Knowles, D [Joshua], Thiele, L., \& Zitzler, E., 2005. A tutorial on the performance assessment of stochastive multiobjective optimizers. Technical report TIK-Report No. 214, Computer Engineering and Networks Laboratory, ETH Zurich.

Lust, T., \& Teghem, J., 2012. The multiobjective multidimensional knapsack problem: a survey and a new approach. International Transactions in Operational Research 19 (4), 495-520.

Lv, Q., Xia X., \& Qian, P., 2006. A parallel ACO approach based on one pheromone matrix. Proceedings of the 5th International Workshop on Ant Colony Optimization and Swarm Intelligence. Lecture Notes in Computer Science. 4150. 332-339.

Mansour, I. B., Alaya, I., \& Moncef, T., 2017a. A Min-Max Tchebycheff Based Local Search Approach for MOMKP. In ICSOFT (pp. 140-150).

Mansour, I. B., Alaya, I., \& Moncef, T., 2017b. Chebyshev-based iterated local search for multi-objective optimization. In 2017 13th IEEE International Conference on Intelligent Computer Communication and Processing (ICCP). 163-170.

Mansour, I. B., Alaya, I., \& Moncef, T., 2019. A gradual weight-based ant colony approach for solving the multiobjective multidimensional knapsack problem. Evolutionary Intelligence, 12(2), 253-272.

Martello S., 1990. Knapsack problems: algorithms and computer implementations. Wiley-Interscience series in discrete mathematics and optimization.

Mora, AM., García-Sánchez, P., Merelo, JJ., \& Castillo, PA., 2013. Pareto-based multi-colony multi-objective ant colony optimization algorithms: an island model proposal. Soft Computing 17.7. 1175-1207.

Pedemonte, M., Nesmachnow, S., \& Cancela, H., 2011. A survey on parallel ant colony optimization. Applied Soft Computing. 11, 5181-5197.

Steuer, R. E., \& Choo, E. U., 1983. An interactive weighted Tchebycheff procedure for multiple objective programming. Mathematical programming, 26(3), 326-344.

Stützle T., 1998. Parallelization strategies for ant colony optimization. Proceedings of the 5th International Conference on Parallel Problem Solving from Nature, Lecture Notes in Computer Science. 1498 722-731.

Stützle, T., \& Hoos, H. H. 2000. MAX-MIN ant system. Future generation computer systems, 16(8), 889-914.

Talbi, EG., Roux, O., Fonlupt, C., \& Robillard, D., 2001. Parallel ant colonies for the quadratic assignement problem, Future Generation Computer Systems 17 (4) 441-449.

Zitzler, E., \& Thiele, L., 1999. Multiobjective Evolutionary Algorithms: A Comparative Case Study and the Strength Pareto Approach. IEEE Transactions on Evolutionary Computation. 3(4): 257-271.

Zouari, W., Alaya, I., \& Moncef, T., 2017. A hybrid ant colony algorithm with a local search for the strongly correlated knapsack problem. In 2017 IEEE/ACS 14th International Conference on Computer Systems and Applications (AICCSA). 527-533. 